\title{
Article
}

\section{Genomic Risk Prediction for Breast Cancer in Older Women}

\author{
Paul Lacaze ${ }^{1,2, *(\mathbb{D}}$, Andrew Bakshi ${ }^{1}$, Moeen Riaz ${ }^{1}$, Suzanne G. Orchard ${ }^{1}$, Jane Tiller ${ }^{1}{ }^{(}$, Johannes T. Neumann ${ }^{1}$, \\ Prudence R. Carr ${ }^{1}{ }^{(D}$, Amit D. Joshi ${ }^{2}$, Yin Cao ${ }^{3}$, Erica T. Warner ${ }^{2}$, Alisa Manning ${ }^{2}$, Tú Nguyen-Dumont ${ }^{4,5}{ }^{1}$, \\ Melissa C. Southey ${ }^{4,5}$, Roger L. Milne ${ }^{4,5,6}$, Leslie Ford ${ }^{7}$, Robert Sebra ${ }^{8}$, Eric Schadt ${ }^{8}$, Lucy Gately ${ }^{9}$, Peter Gibbs ${ }^{9}$, \\ Bryony A. Thompson ${ }^{10}$, Finlay A. Macrae ${ }^{10}$, Paul James ${ }^{10}$, Ingrid Winship ${ }^{10}$, Catriona McLean ${ }^{11}$, \\ John R. Zalcberg ${ }^{1}$, Robyn L. Woods ${ }^{1}$, Andrew T. Chan ${ }^{2}$, Anne M. Murray ${ }^{12}$ and John J. McNeil ${ }^{1}{ }^{1}$
}

1 Department of Epidemiology and Preventive Medicine, School of Public Health and Preventive Medicine, Monash University, Melbourne, VIC 3004, Australia; Andrew.Bakshi1@monash.edu (A.B.); Moeen.Riaz@monash.edu (M.R.); Suzanne.Orchard@monash.edu (S.G.O.); jane.tiller@monash.edu (J.T.); Johannes.Neumann@monash.edu (J.T.N.); Prue.Carr@monash.edu (P.R.C.); john.zalcberg@monash.edu (J.R.Z.); Robyn.Woods@monash.edu (R.L.W.); john.mcneil@monash.edu (J.J.M.)

2 Clinical and Translational Epidemiology Unit, MGH Cancer Center, Massachusetts General Hospital and Harvard Medical School, Boston, MA 02108, USA; ADJOSHI@mgh.harvard.edu (A.D.J.); EWarner@mgh.harvard.edu (E.T.W.); AKMANNING@mgh.harvard.edu (A.M.); achan@mgh.harvard.edu (A.T.C.)

3 Alvin J. Siteman Cancer Center, Division of Public Health Sciences, Department of Surgery, Washington University School of Medicine, St Louis, MO 63110, USA; yin.cao@wustl.edu

4 Precision Medicine, School of Clinical Sciences at Monash Health, Monash University, Melbourne, VIC 3168, Australia; Tu.Nguyen-Dumont@monash.edu (T.N.-D.);

Melissa.Southey@monash.edu (M.C.S.); Roger.Milne@cancervic.org.au (R.L.M.)

5 Department of Clinical Pathology, University of Melbourne, Melbourne, VIC 3010, Australia

6 Cancer Epidemiology Division, Cancer Council Victoria, Melbourne, VIC 3004, Australia

7 Division of Cancer Prevention, National Cancer Institute, Rockville, MD 20892, USA; fordl@mail.nih.gov

8 Department of Genetics and Genomic Sciences, Icahn School of Medicine at Mount Sinai,

Citation: Lacaze, P.; Bakshi, A.; Riaz, M.; Orchard, S.G.; Tiller, J.; Neumann, J.T.; Carr, P.R.; Joshi, A.D.; Cao, Y.; Warner, E.T.; et al. Genomic Risk Prediction for Breast Cancer in Older Women. Cancers 2021, 13, 3533. https://doi.org/10.3390/ cancers 13143533

Academic Editor: Antonio Russo

Received: 11 June 2021

Accepted: 12 July 2021

Published: 14 July 2021

Publisher's Note: MDPI stays neutral with regard to jurisdictional claims in published maps and institutional affiliations. New York, NY 10029, USA; robert.sebra@mssm.edu (R.S.); eric.schadt@mssm.edu (E.S.)

9 Personalised Oncology Division, Walter and Eliza Hall Institute Medical Research, Faculty of Medicine, University of Melbourne, Melbourne, VIC 3052, Australia; lucyjgately@hotmail.com (L.G.); Peter.Gibbs@mh.org.au (P.G.)

10 Department of Genomic Medicine, Royal Melbourne Hospital, University of Melbourne, Parkville, Melbourne, VIC 3050, Australia; Bryony.Thompson@mh.org.au (B.A.T.);

Finlay.Macrae@mh.org.au (F.A.M.); Paul.James@petermac.org (P.J.); ingrid.winship@mh.org.au (I.W.)

11 Department of Anatomical Pathology, Alfred Hospital, Melbourne, VIC 3004, Australia; C.McLean@alfred.org.au

12 Berman Center for Outcomes and Clinical Research, Hennepin Healthcare Research Institute, Hennepin Healthcare, University of Minnesota, Minneapolis, MN 55404, USA; AMurray@bermancenter.org

* Correspondence: paul.lacaze@monash.edu; Tel.: +61-3-9903-0412

Simple Summary: We designed a study specifically to assess the performance of genomic risk prediction for breast cancer $(\mathrm{BC})$ in older women aged $\geq 70$ years. We assessed the effects of a polygenic risk score (PRS) for BC and rare pathogenic variants (PVs) in BC susceptibility genes, on incident $\mathrm{BC}$ risk in a prospective cohort of 6339 older women (mean age 75 years). During a median follow-up time of 4.7 years, the PRS was an independent predictor of incident BC risk, with women in the top quintile of the PRS distribution having over two-fold higher incident BC risk than women in the lowest quintile. Among 41 carriers of PVs in BC susceptibility genes, we observed no incident BC diagnoses. Our study demonstrates that a PRS still predicts incident BC risk in women aged 70 years and older, suggesting the potential clinical utility of the PRS extends to this older age group.

Abstract: Genomic risk prediction models for breast cancer (BC) have been predominantly developed with data from women aged 40-69 years. Prospective studies of older women aged $\geq 70$ years have been limited. We assessed the effect of a 313-variant polygenic risk score (PRS) for BC in 6339 older women aged $\geq 70$ years (mean age 75 years) enrolled into the ASPREE trial, a randomized double-blind placebo-controlled clinical trial investigating the effect of daily $100 \mathrm{mg}$ aspirin on disability-free survival. We evaluated incident BC diagnoses over a median follow-up time of 
4.7 years. A multivariable Cox regression model including conventional BC risk factors was applied to prospective data, and re-evaluated after adding the PRS. We also assessed the association of rare pathogenic variants (PVs) in BC susceptibility genes (BRCA1/BRCA2/PALB2/CHEK2/ATM). The PRS, as a continuous variable, was an independent predictor of incident $B C$ (hazard ratio (HR) per standard deviation $(\mathrm{SD})=1.4,95 \%$ confidence interval (CI) 1.3-1.6) and hormone receptor (ER/PR)positive disease ( $\mathrm{HR}=1.5(\mathrm{CI} 1.2-1.9))$. Women in the top quintile of the PRS distribution had over two-fold higher risk of $\mathrm{BC}$ than women in the lowest quintile ( $\mathrm{HR}=2.2$ (CI 1.2-3.9)). The concordance index of the model without the PRS was 0.62 (95\% CI 0.56-0.68), which improved after addition of the PRS to 0.65 (95\% CI 0.59-0.71). Among 41 (0.6\%) carriers of PVs in BC susceptibility genes, we observed no incident BC diagnoses. Our study demonstrates that a PRS predicts incident BC risk in women aged 70 years and older, suggesting potential clinical utility extends to this older age group.

Keywords: genomics; breast cancer; risk prediction; polygenic risk score; germline

\section{Introduction}

Breast cancer $(\mathrm{BC})$ risk prediction models may be improved by including genomic risk scores. A polygenic risk score (PRS) aggregates the effect of many common BC risk-associated variants into a single measure [1-4]. Common BC risk-associated genetic variants used in a PRS, together, are estimated to account for $18 \%$ of familial $\mathrm{BC}$ risk [5]. Predictive performance of a PRS for BC has mostly been assessed in women aged 40-69 years [1,3,6-10]. PRS performance, in terms of risk prediction in older women (aged $\geq 70$ years), is unclear, despite a high proportion of $\mathrm{BC}$ diagnoses occurring in this age group. It is also unclear whether the predictive performance of a PRS for BC attenuates with age. Given the emerging clinical utility of PRS for BC risk prediction and stratification, this requires further assessment.

A small proportion of women $(<5 \%)$ carry rare pathogenic variants $(\mathrm{PVs})$ in $\mathrm{BC}$ predisposition genes, including BRCA1, BRCA2, PALB2, CHEK2 and ATM [11]. Rare PVs can be detected by predictive clinical genetic testing and are of high clinical significance [12,13], predisposing women to BC earlier in life [6-8,11]. Rare PVs account for $\sim 25 \%$ of familial BC risk [14]. Recent studies have suggested that the increase in $B C$ risk associated with rare PVs in high-risk genes (e.g., $B R C A 1, B R C A 2$ ) may decrease with increasing age $[6,8,10,12,13]$. If rare PVs have age-dependent effects on $B C$ risk, this may have important clinical implications for the appropriateness of offering predictive genetic testing to older people.

Many clinical studies have measured the association between rare PVs and BC risk [6-8,11]. More recently, risk conferred by a 313-variant PRS for BC has been measured in meta-analysis of ten studies, with no evidence of age-related attenuation in the predictive performance of the PRS reported [1]. The same PRS has been assessed across several subsequent PRS validation studies [2-4]. However, participant numbers aged $\geq 70$ years were limited. Here, we evaluate the predictive performance of (i) a PRS for BC and (ii) rare PVs in a prospective cohort of 6339 women aged $\geq 70$ years.

\section{Materials and Methods}

\subsection{Study Sample}

The study sample comprised female participants of the ASPirin in Reducing Events in the Elderly (ASPREE) trial—a randomized, placebo-controlled, clinical trial investigating the effect of daily 100mg aspirin on disability-free survival [15-17]. Study design [18,19], recruitment [20], and baseline characteristics [21] have been published previously. The trial recruited 19,114 individuals from Australia $(n=16,703)$ and the United States $(n=2411)$ aged 70 years or older ( $\geq 65$ years for US ethnic minorities), who, at enrolment, were free from diagnosed cardiovascular disease events, dementia, physical disability and lifethreatening cancer diagnoses. Biospecimens and consent for genetic analysis were obtained from 14,576 participants. The median follow-up time (randomization period) was 4.7 years 
(interquartile range 2.1 years). The study received approvals from the Alfred Hospital Research Ethics Committee (Project 390/15) and is registered (NCT01038583). All participants provided written informed consent for genetic research. The final analysis for this study was conducted on 6339 female ASPREE participants from Australia aged $\geq 70$ years, for whom both genome-wide genotyping and targeted sequencing data were available.

\subsection{Genome-Wide Genotyping and Polygenic Risk Score}

DNA samples were genotyped using the Axiom 2.0 Precision Medicine Diversity Research Array (Thermo Fisher Scientific (TFS), Waltham, MA, USA) following standard protocols. Only participants with European genetic ancestry ( $>95 \%$ of female participants) were included, to mitigate population stratification bias. Genetic ancestry was defined using principal component analysis (PCA) based on the 1000 Genomes reference population, with participants outside of the Non-Finnish European ancestry cluster excluded (Figure S1) [22]. Imputation was performed using the TopMED Server (European samples) [23]. After variants with low imputation quality scores $\left(r^{2}<0.3\right)$ were excluded, a PRS was calculated based on the 313-variant score previously described [1], using genotypes for the remaining 271 variants (for a list of the 313 variants in the PRS, indicating the 271 variants included in the final analysis, see Table S8). Using Plink version 1.9, we calculated the PRS for each individual as the weighted sum of the effect size for the number of risk alleles at each variant [1].

\subsection{Targeted Gene Panel Sequencing}

Our custom gene panel [24] included BC predisposition genes that are incorporated into the Breast and Ovarian Analysis of Disease Incidence and Carrier Estimation Algorithm (BOADICEA) - BRCA1, BRCA2, PALB2, CHEK2 and ATM [11]. Following standard protocols, DNA was extracted and sequenced using the S5TM XL system (Thermo Fisher Scientific (TFS), Waltham, MA, USA), to average $200 \times$ depth. Variants with a 'pathogenic' or 'likely pathogenic' ClinVar annotation [25] and/or high-confidence predicted loss-offunction in coding regions [26] were curated following ACMG/AMP Standards and Guidelines for the Interpretation of Sequence Variants [27], including review by two or more laboratory scientists and a clinical geneticist. Analysis was restricted to single nucleotide variants and small insertions/deletions.

\subsection{Endpoints}

The study's primary endpoint was invasive breast cancer (BC), which included incident invasive $B C$ diagnosed during the ASPREE trial; this was adjudicated by an expert panel using histopathology, metastasis imaging or other clinical evidence [28]. Age at diagnosis of prevalent $B C$ was self-reported as before or after 50 years.

\subsection{Statistical Analysis}

After excluding female participants with a history of prevalent BC at enrolment, multivariable Cox proportional hazards regression was used to evaluate the association between PRS with incident $B C$ by estimating the hazard ratio (HR) per standard deviation (SD) of the PRS, after adjusting for BC family history (first-degree blood relatives), treatment (aspirin/placebo), age at enrolment, number of children, alcohol consumption, body mass index (BMI) at enrolment, and use of estrogen or estrogen/progesterone hormone replacement therapy (HRT) at enrolment. Alcohol consumption was categorised into three groupings: none (no current consumption); low ( $<3$ drinking days per week); and high ( $\geq 3$ drinking days per week). Interaction between PRS and aspirin treatment was tested independently alongside the complete set of covariates. BMI and number of children were standardised to mean $=0$ (SD 1).

In a separate model, PRS was categorized into three quintile-based distribution groups-low (0-20\%, Q1), moderate-risk (21-80\%, Q2-4), and high-risk (81-100\%, Q5). R package pec v2019.11.03 was used for BC-free survival prediction. Net reclassification improvement (NRI) was calculated using R package nricens v1.6 with $1 \%$ and $3 \%$ cutoff values 
for predicting increased and decreased risk categories. We calculated cumulative incidence estimates for each PRS group (multivariate adjusted), treating death as a competing risk.

Logistic regression was used to assess associations with prevalent breast cancer, including family history and the presence of rare PVs as covariates. We further stratified by diagnosis age $(<50,50+)$, and included number of children in the model. We used variance inflation factor (VIF) to assess the independence of predictors, and measured the discriminative ability of the PRS using concordance index and area under the receiver operating characteristic curve (AUC). Goodness-of-fit for the logistic regression was assessed using the Hosmer-Lemeshow (HL) test and the Tail-Based Max-test-statistic (TBM) [29]. DeLong's test was used to compare between two correlated ROC curves [30,31]. We calculated PV ORs for prevalent BC for each gene and all five genes combined. Analyses were performed using R v3.6.1.

\section{Results}

\subsection{Baseline Characteristics}

The mean age of the 6339 female participants of European genetic ancestry was 75.1 years at time of enrolment, with $14 \%$ aged $>80$ years, and $31 \%$ current or former smokers (Table 1). The mean BMI was $28.0 \mathrm{~kg} / \mathrm{m}^{2}, 75 \%$ were current alcohol consumers and $13 \%$ had a family history of BC in a first-degree blood relative. At baseline, $533(8.4 \%)$ participants were taking HRT (either estrogen alone or with progesterone). Six $(0.2 \%)$ were taking progesterone-only preparations. Prevalent BC was reported by $475(7.6 \%)$ participants, of which $60(1 \%)$ were diagnosed before the age of 50 years.

Table 1. Characteristics of the study population.

\begin{tabular}{|c|c|c|c|c|}
\hline Characteristics & Total & $\begin{array}{c}\text { Low-Risk PRS } \\
\text { (Q1) }\end{array}$ & $\begin{array}{c}\text { Moderate-Risk PRS } \\
\text { (Q2-4) }\end{array}$ & $\begin{array}{c}\text { High-Risk PRS } \\
\text { (Q5) }\end{array}$ \\
\hline Participants & $n=6339$ & $n=1268$ & $n=3803$ & $n=1268$ \\
\hline Sex $=$ Female $(\%)$ & $6339(100)$ & $1268(100)$ & $3803(100)$ & $1268(100)$ \\
\hline Mean Age at Enrolment, Years & 75.1 & 75.1 & 75.1 & 75.2 \\
\hline \multicolumn{5}{|l|}{ Age Group, Years (\%) } \\
\hline $70-74$ & $3825(60.3)$ & $778(61.4)$ & $2287(60.1)$ & $760(59.9)$ \\
\hline $75-79$ & 1599 (25.5) & $304(24.0)$ & $975(25.6)$ & $320(25.2)$ \\
\hline $80-84$ & $706(11.1)$ & $136(10.7)$ & $429(11.3)$ & $141(11.1)$ \\
\hline $85+$ & $211(3.3)$ & $50(3.9)$ & $112(2.9)$ & $47(3.7)$ \\
\hline Current or Former Smoker (\%) & $1974(31.1)$ & $391(30.8)$ & $1195(31.4)$ & $388(30.6)$ \\
\hline Diabetes (\%) & $497(7.8)$ & $97(7.6)$ & $289(7.6)$ & $111(8.8)$ \\
\hline Randomized to Aspirin (\%) & $3170(50.0)$ & $630(49.7)$ & $1898(49.9)$ & $642(50.6)$ \\
\hline Body Mass Index kg/m² ((mean) (SD)) & $28.03(5.09)$ & $28.02(5.02)$ & $28.05(5.10)$ & $28.00(5.13)$ \\
\hline Current Alcohol Consumption (\%) & $4730(74.6)$ & $941(74.2)$ & $2850(74.9)$ & $939(74.1)$ \\
\hline Hormone Replacement Therapy * & $533(8.4)$ & $103(8.1)$ & $321(8.4)$ & $109(8.6)$ \\
\hline Progesterone-Only HRT & $6(0.2)$ & $1(0.1)$ & $3(0.1)$ & $2(0.2)$ \\
\hline Family History of Breast Cancer $(\%) \ddagger$ & $850(13.4)$ & $135(10.6)$ & $500(13.1)$ & $215(17.0)$ \\
\hline \multicolumn{5}{|l|}{ Prevalent Breast Cancer } \\
\hline Cases & 475 & 47 & 288 & 140 \\
\hline Diagnosed $<49$ Years & 60 & 6 & 39 & 15 \\
\hline Diagnosed 50+ Years & 415 & 41 & 249 & 125 \\
\hline \multicolumn{5}{|l|}{ Incident Breast Cancer II } \\
\hline Cases & 129 & 21 & 66 & 42 \\
\hline Polygenic Risk Score (mean (SD)) & $0.1(0.53)$ & $-0.93(0.26)$ & $-0.13(0.27)$ & $0.69(0.28)$ \\
\hline
\end{tabular}

PRS = Polygenic risk score, $\mathrm{Q}=$ Quintile, HRT = Hormone replacement therapy. * Estrogen alone or in combination with progesterone; II Non-metastatic and metastatic events; $\ddagger$ Family history in first-degree blood relative (mother, sibling or child). 


\subsection{PRS and Rare Pathogenic Variants}

The PRS showed a normal distribution in the study sample with mean -0.13 (SD 0.58) before standardization (Figure S2A), which was scaled to a mean of 0 (SD 1) for subsequent analyses. Forty-two rare pathogenic or likely pathogenic variants (PVs) passed our variant curation protocol (Table S1) across $41(0.6 \%)$ participants (one participant had two PVs detected, one each in $B R C A 2$ and CHEK2). We identified participants with PVs in the BRCA1 $(n=3), B R C A 2(n=10)$, PALB2 $(n=6)$, CHEK2 $(n=7)$ and ATM $(n=16)$ genes. Of these participants, $20 \%$ reported a family history of BC in a first-degree blood relative at enrolment.

\subsection{Incident Breast Cancer Risk}

After excluding the 475 female participants with prevalent $B C$ at enrolment, during median follow-up (4.7 years/participant), 110 women had incident $B C$. None of these women had rare PVs in the BRCA1, BRCA2, PALB2, CHEK2, and ATM genes. In the multivariable Cox model, conventional $\mathrm{BC}$ risk factors, including family history of $\mathrm{BC}$, number of children, alcohol consumption and estrogen HRT were associated with risk of incident BC (Table 2).

Table 2. Association of a polygenic risk score (PRS) with incident breast cancer (BC) risk in 6339 older women. A multivariable Cox proportional hazards regression model was used to evaluate the association between PRS as a continuous or categorical variable with incident BC ( $n=110$ cases), after adjusting for family history of BC (first-degree blood relatives), treatment (aspirin/placebo), age at enrolment, number of children, alcohol use, BMI at enrolment and use of hormone replacement therapy (HRT) at enrolment.

\begin{tabular}{|c|c|c|c|c|c|c|}
\hline \multirow{2}{*}{ Variable } & \multicolumn{3}{|c|}{ PRS as Continuous Variable } & \multicolumn{3}{|c|}{ PRS as Categorical Variable } \\
\hline & Hazard Ratio & $95 \%$ CI & $p$-Value & Hazard Ratio & $95 \%$ CI & $p$-Value \\
\hline $\begin{array}{c}\text { Polygenic Score } \\
\text { (per standard deviation) }\end{array}$ & 1.43 & $(1.18 ; 1.73)$ & $<0.001$ & & & \\
\hline Low PRS (Q1) & & & & \multicolumn{3}{|c|}{ Reference } \\
\hline Moderate PRS (Q2,3,4) & & & & 1.16 & $(0.68 ; 2.00)$ & 0.58 \\
\hline High PRS (Q5) & & & & 2.16 & $(1.21 ; 3.86)$ & 0.009 \\
\hline $\begin{array}{l}\text { Pathogenic Variants } \\
\quad(n=41 \text { carriers })\end{array}$ & \multicolumn{3}{|c|}{ No incident events } & \multicolumn{3}{|c|}{ No incident events } \\
\hline Family History of Breast Cancer * $(\mathrm{Y} / \mathrm{N})$ & 1.81 & $(1.15 ; 2.85)$ & 0.01 & 1.83 & $(1.16 ; 2.88)$ & 0.009 \\
\hline Age at Enrolment & 0.97 & $(0.92 ; 1.02)$ & 0.21 & 0.97 & $(0.92 ; 1.02)$ & 0.22 \\
\hline Treatment (Aspirin) & 1.16 & $(0.80 ; 1.69)$ & 0.44 & 1.15 & $(0.79 ; 1.68)$ & 0.45 \\
\hline Number of Children & 0.81 & $(0.66 ; 0.99)$ & 0.04 & 0.81 & $(0.66 ; 0.99)$ & 0.04 \\
\hline Body Mass Index (kg/m² (mean) SD) & 1.14 & $(0.95 ; 1.37)$ & 0.17 & 1.14 & $(0.95 ; 1.37)$ & 0.15 \\
\hline Alcohol (None) & \multicolumn{3}{|c|}{ Reference } & \multicolumn{3}{|c|}{ Reference } \\
\hline Alcohol (Low) & 1.16 & $(0.68 ; 1.97)$ & 0.59 & 1.16 & $(0.68 ; 1.98)$ & 0.58 \\
\hline Alcohol (High) & 1.70 & $(1.01 ; 2.85)$ & 0.04 & 1.70 & $(1.02 ; 2.86)$ & 0.04 \\
\hline $\mathrm{HRT} \ddagger(\mathrm{Y} / \mathrm{N})$ & 1.54 & $(0.88 ; 2.71)$ & 0.13 & 1.51 & $(0.86 ; 2.65)$ & 0.15 \\
\hline
\end{tabular}

$\mathrm{BMI}=$ Body mass index, HRT $=$ Hormone replacement therapy, PRS = Polygenic risk score, $\mathrm{CI}=$ Confidence Interval, $\mathrm{SD}=\mathrm{Standard}$ deviation. * Family history in first-degree blood relative (mother, sibling or child); $\ddagger$ Estrogen alone or in combination with progesterone.

The PRS, as a continuous variable in the same model, was an independent predictor of incident BC, with a HR of 1.43 (95\% confidence interval (CI) 1.18 to $1.73, p<0.001)$ per SD (Table 2, Figure 1), after adjustment for covariates. The PRS was also an independent predictor of incident hormone receptor (ER/PR)-positive BC (HR = 1.5 (CI 1.2-1.9), $p<0.001$ ), after adjustment for covariates. The VIF for each term in the multivariable model was less than 1.1, indicating the independence of the predictors. The concordance index of the 
model without the PRS was 0.62 (95\% CI 0.56 to 0.68 ), which improved after addition of the PRS to 0.65 (95\% CI 0.59 to 0.71 ). We found no evidence of an interaction between aspirin treatment and the PRS.

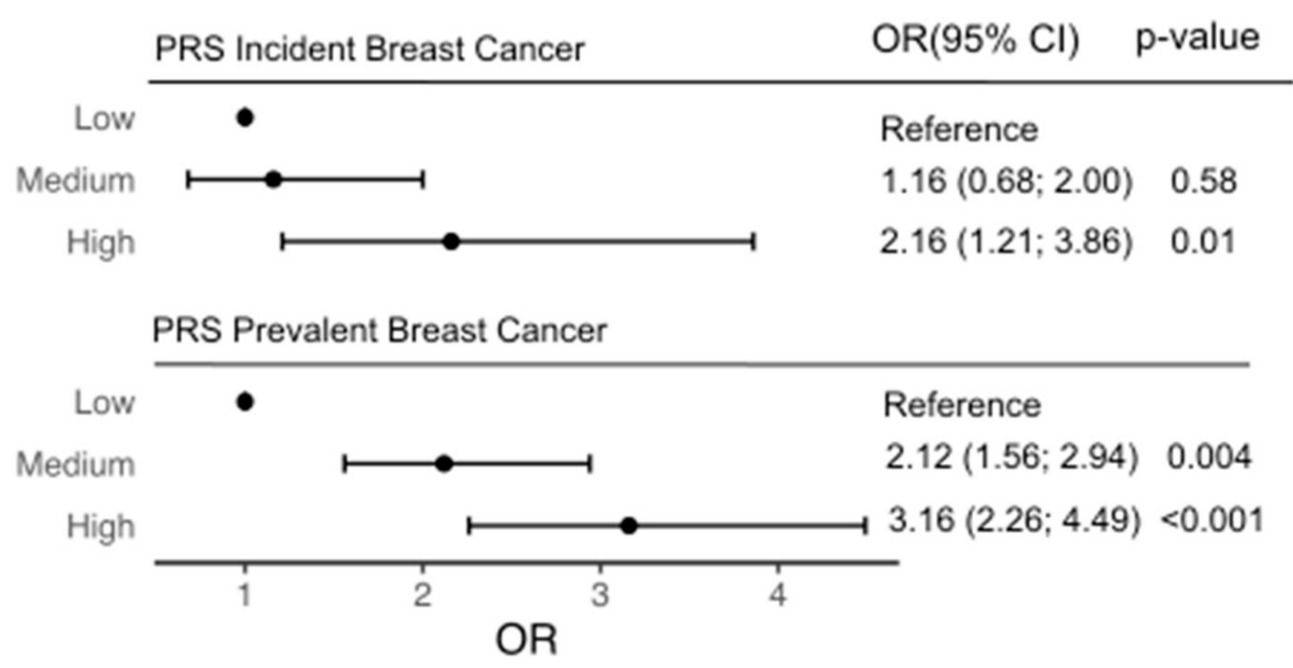

Figure 1. Association of a polygenic risk score (PRS) with incident and prevalent breast cancer (BC) risk in 6339 older women. We evaluated incident $\mathrm{BC}$ diagnoses over a median follow-up of 4.7 years and prevalent BC diagnosed pre-enrolment (self-reported). A multivariable Cox regression model including conventional risk factors examined the association between incident $\mathrm{BC}$ risk and the PRS as a categorical variable by quintiles $(\mathrm{Q})$ of the distribution (low- (Q1), medium- (Q2-4), high- (Q5) risk groups), adjusting for family history of BC (first-degree blood relatives), treatment (aspirin/placebo), age at enrolment, number of children, alcohol consumption, body mass index (BMI) at enrolment, and use of estrogen or estrogen/progesterone hormone replacement therapy (HRT) at enrolment. Logistic regression examined associations with prevalent BC, adjusting for family history of BC (first-degree blood relatives) and pathogenic variants in BC-associated genes, represented by odds ratio (OR).

We categorized the PRS into low- (Q1), moderate- (Q2-4) and high-risk (Q5) groups to consider PRS effect on incident BC. When using Q1 as a reference, participants in the high-risk PRS group had a significantly higher risk of developing incident $\mathrm{BC}$ compared to women in the low-risk PRS group ( $\mathrm{HR}=2.16$ (95\% CI 1.21 to 3.86), $p<0.01$ ) (Table 2, Figure 2). The competing risk model showed that individuals in Q5 (the high-risk group) had higher cumulative incidence than those in Q1 (the low-risk group) and Q2-4 (the moderate-risk group) (Figure 2). Participants in the moderate- and low-risk groups did not have significantly different risks of incident BC. The calibration plot for the incident risk model (Figure S2B) illustrates high concordance between the predicted and observed events. Net reclassification analysis had point estimates of 0.15 (95\% CI $0.03 ; 0.24)$ for combined change, with NRI+ of $0.09(95 \% \mathrm{CI}-0.02 ; 0.13)$ and NRI- of 0.05 (95\% CI 0.02; 0.08). Reclassification of cases and controls is shown in Table S2.

Histopathology was available for 103 incident BC cases (Table S3). The PRS was found to be a significant predictor of (ER+/PR+) disease (HR $=1.53$ per SD ( $95 \%$ CI 1.22 to 1.91$)$ ), $p<0.001, n=79$ ).

\subsection{Prevalent Breast Cancer}

Of the 41 participants with PVs, 11 (27.5\%) reported prevalent BC at baseline, compared with $7.5 \%(475 / 6339)$ in all female participants, giving an estimated OR of $4.69(95 \%$ CI 2.21 to 9.27, $p<0.001$ ) for PVs grouped across all five genes (Table S4). The OR estimate for PVs in BRCA1 and BRCA2 (OR = 6.09 (95\% CI 1.77 to 19.08)) was higher than PVs in the other genes $(A T M / P A L B / C H E C K 2)(\mathrm{OR}=3.33(95 \% \mathrm{CI} 1.19$ to 7.92$), p=0.011)$. Per gene ORs are reported in Table S5, but are limited by small carrier numbers. 


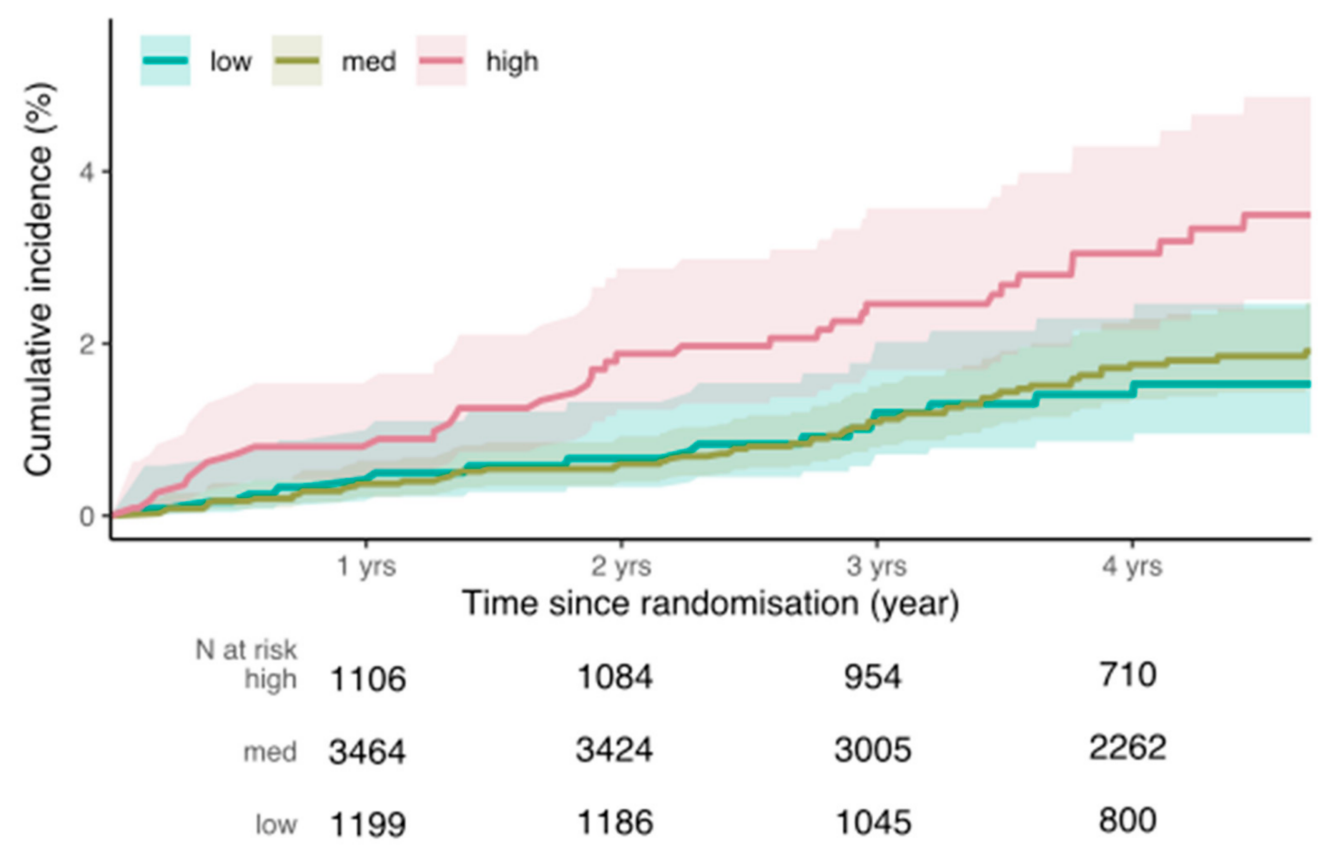

Figure 2. Competing risk survival curves for incident breast cancer according to PRS groups. The PRS distribution was categorized by quintiles (Q) of the distribution into three groups: low-risk (Q1, green), medium-risk (Q2-4, brown) and high-risk (Q5, red). Competing risk estimates of the cumulative incidence were calculated for each group, adjusting for the following covariates: family history of BC (first-degree blood relatives), treatment (aspirin/placebo), age at enrolment, number of children, alcohol consumption, body mass index (BMI) at enrolment, and use of estrogen or estrogen/progesterone hormone replacement therapy (HRT) at enrolment.

In sub-group analysis, when stratifying prevalent BC cases by diagnosis age (before or after 50 years), the OR estimate for having a PV was higher for early onset BC risk (diagnosed $<50$ years, $\mathrm{OR}=9.79$ (CI 2.29 to 28.87), $p=0.02$ ) than for later-onset BC risk (diagnosed $>50$ years, $\mathrm{OR}=3.91$ (CI 1.64 to 8.31$), p=0.02$ ) (Table S6).

The PRS as a continuous variable was associated with prevalent BC after controlling for covariates in the model (OR = 1.47 per SD (95\% CI 1.34 to 1.61$), p<0.001)$. The HL and TBM tests did not indicate a lack of goodness-of-fit $(p>0.05)$. The AUC for the model with the PRS (AUC $=0.62$ (0.59-0.65), Figure S2C) was improved relative to the model including family history of BC only $(\mathrm{AUC}=0.53(0.52-0.55)$, Figure S2D) $(p<0.01)$. When considering the PRS as a categorical variable, participants in the high-risk group had a significantly higher BC risk compared with the low-risk group (OR $=3.16$ (95\% CI 2.26 to $4.49), p<0.001)$. Participants in the moderate-risk group also had higher BC risk versus the low-risk group $(\mathrm{OR}=2.12(95 \%$ CI 1.56 to 2.94$), p<0.001)$.

\subsection{Modification of BC Risk by PRS in Rare PV Carriers}

Eleven participants with PVs reported prevalent BC, and 29 with PVs reported no prevalent BC. We hypothesized that individuals with PVs and a history of BC (affected) may have a higher PRS, on average, than those with PVs but without BC (unaffected), as suggested by previous $B R C A 1 / B R C A 2$ studies [32]. However, we observed no evidence of over-representation of affected or unaffected carriers between low-, moderate- or high-risk PRS groups (Chi-squared $\chi^{2}=1.97, \mathrm{df}=2, p=0.37$ ) (Figure 3, Table S7). 


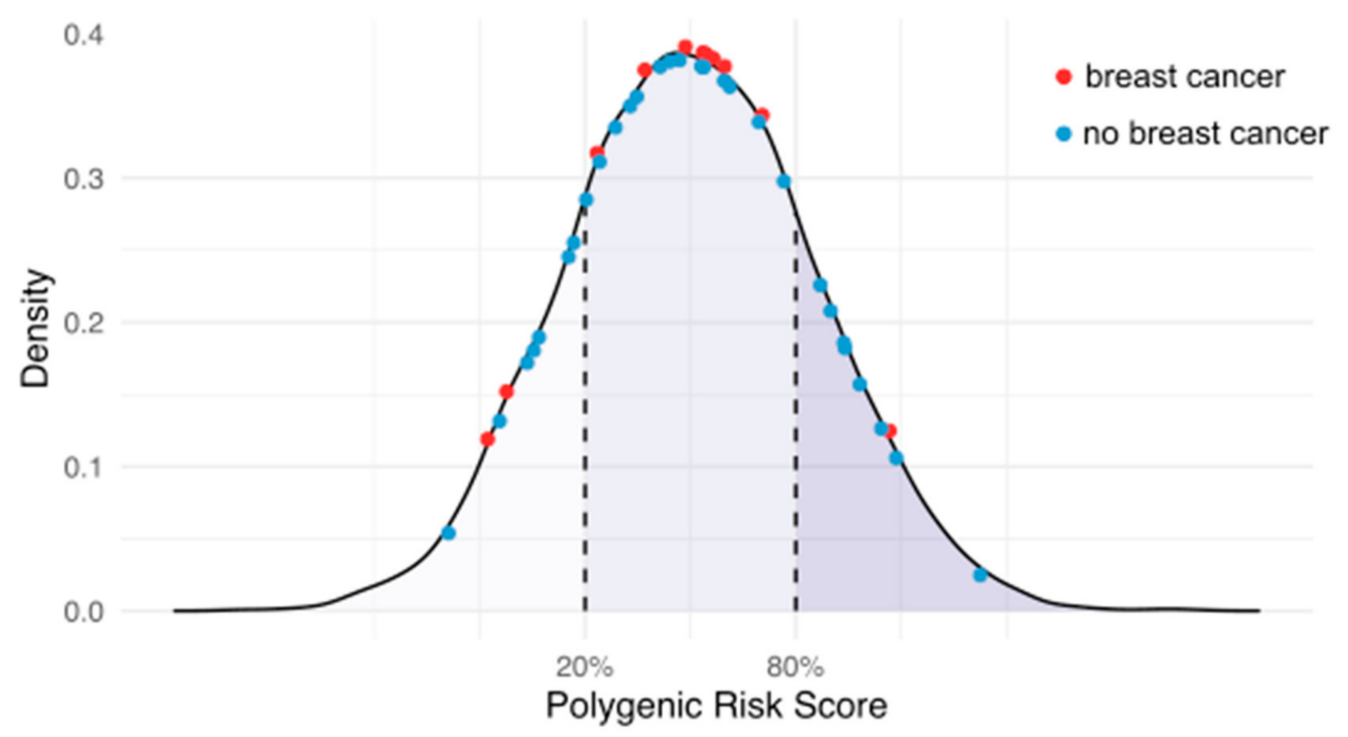

Figure 3. PRS distribution of female pathogenic variant (PV) carriers who were affected (red) or unaffected (blue) by prevalent BC. Density represents the proportion of individuals in each PRS group (low/medium/high). The PRS is distributed normally according to scaled PRS score (mean 0, standard deviation 1). PV carriers are highlighted across the PRS distribution.

\section{Discussion}

In this study, we assessed the performance of a 313-variant PRS for BC and rare pathogenic variants in $\mathrm{BC}$-susceptibility genes for risk prediction of $\mathrm{BC}$ in older women. We found that the PRS was a significant predictor of incident $B C$ risk, when considered as both a continuous (per SD) or categorical (low, moderate and high-risk groups) variable. Net reclassification was improved after addition of the PRS to a model composed of traditional BC risk factors. Older women in the highest quintile of the PRS distribution had over two-fold higher risk of incident BC than those in the lowest quintile. The PRS was also associated with incident hormone receptor positive (ER+/PR+) disease specifically, and with prevalent $\mathrm{BC}$. The emerging clinical utility of PRS for $\mathrm{BC}$ risk prediction and risk stratification, previously demonstrated in women aged 40-69 years [1-4], therefore likely extends to older women.

The PRS effect $(\mathrm{HR}=1.4$ per $\mathrm{SD})$ was similar to that reported in population-based studies of younger women $[1,3,9,10]$, including a recent meta-analysis measuring the same PRS used in our study across ten prospective studies of younger women where ORs ranged from 1.48 to 1.75 across participants of all ages [1]. Subsequent validation studies of the same PRS in other cohorts found similar ORs to our study ( 1.4) [2-4]. Notably, the average age at enrolment in the ASPREE female population (75 years) is over 15 years older than most other prior population-based studies, including the UK Biobank population, where average enrolment age was 58 years [33]. Yet, similar HRs for the PRS were observed between ASPREE and these prior studies. This suggests that the predictive performance of the PRS does not attenuate with age, in women over 70 years.

No incident $\mathrm{BC}$ diagnoses were observed in 41 women with rare PVs, despite the high expected $\mathrm{BC}$ risk conferred by these PVs (e.g., average cumulative risk to age 70 years of $50-70 \%$ for $B R C A 1 / B R C A 2 \mathrm{PVs}$ ) [8]. This challenges the clinical value of predictive genetic testing for $B C$ risk by sequencing of these genes alone in women aged $\geq 70$ years. However, genetic testing for $\mathrm{BC}$ in older women can trigger cascade family testing, which has benefits including risk management, early detection, and/or prevention of cancer in younger family members. We also observed no incident ovarian cancer diagnoses in the $41 \mathrm{PV}$ carriers.

Retrospective data suggested a higher risk of BC in individuals with a PV compared with those without $(\mathrm{OR}=4.7)$ when all PVs across all genes were combined into a single group. This reflects the effect of PVs earlier in life. However, women with PVs diagnosed 
with cancer earlier in life are less likely to have been ascertained by our study, because they either died from cancer before the enrolment age or were too unwell to enrol in the ASPREE trial due to a current or recent cancer diagnosis. This has likely resulted the healthy selection bias that can often occur in older survivor cohorts [34]. Thus, risk estimates observed in PV carriers ascertained in our study (and the ORs and CIs reported for associations between the PRS/PVs and prevalent cases) must be interpreted with caution.

In response to recent studies reporting that an individual's PRS may modify the penetrance of rare PVs in the BRCA1 and BRCA2 genes [9,10,32,35], we sought evidence of risk modification by the PRS in PV carriers affected and unaffected by prevalent $B C$ in our study. We observed no evidence of either a higher PRS in females with a PV and affected by BC, or conversely a protective effect of a lower PRS in unaffected PV carriers (Figure 3). However, we acknowledge our analysis was limited by a relatively small number of PV carriers. Further studies are needed to investigate this more rigorously.

Clinically, it is notable that most incident BC cases in our study had favourable prognoses (e.g., hormone receptor positive). This raises the possibility that genomic risk prediction for $\mathrm{BC}$ in older women may have limited impact for improving survival, and that this impact must be balanced against potential overdiagnosis/overtreatment risks in this older demographic.

Key strengths of our study include the well-characterised, older study population (median age after follow-up: 78 years) followed prospectively, with all incident BC diagnoses adjudicated by an expert panel. Most previous studies of genetic risk scores for BC have examined younger cohorts, some selected for family history.

Limitations of our study include the unavailability of some phenotypic and clinical risk factors associated with $B C$, such as mammographic density, reproductive factors (e.g., age at menarche, menopause, first birth) and hormonal factors beyond HRT use (e.g., oral contraceptive use) [36]. Our clinical risk factor model might be improved with these additional factors. Some participants may have undergone risk-reducing bilateral prophylactic mastectomy or bilateral salpingo-oophorectomy prior to enrolment, to reduce $\mathrm{BC} / \mathrm{OC}$ risk. The relatively small number of PVs detected $(n=41)$ necessitated grouping of PVs across genes to calculate averaged risk, despite known differences in the magnitude of risk conferred by PVs in different genes [12,13]. Prevalent BC (pre-enrolment) was self-reported (without specific diagnosis age) and not verified through supporting documentation, potentially causing over-estimation of prevalent BC events. Our study involved only participants of European genetic ancestry, meaning results may not be generalisable to other populations.

\section{Conclusions}

In conclusion, we demonstrate that the predictive value of a PRS for BC extends to older women, with no evidence of age-related attenuation in predictive performance after the age of 70 years. Our study has clinical implications for the use and interpretation of polygenic risk prediction of $\mathrm{BC}$ across the female lifespan.

Supplementary Materials: The following are available online at https: / www.mdpi.com/article / 10.3390 / cancers13143533/s1, Figure S1: Principal component (PC) analysis of the ASPREE cohort compared with the 1000 Genome Project, Figure S2: Distribution of the PRS, Calibration of the Risk Model (Incident BC), and Area Under the Curve for the Logistic Regression Model (Prevalent BC), Table S1: Table of pathogenic variants (PVs) detected in breast cancer susceptibility genes (BRCA1, BRCA2, PALB2, CHECK2, ATM) in 6339 older women, Table S2: Categorical net reclassification improvement after adding Polygenic Risk Score to the conventional model to predict 4.7-years risk of breast cancer, Table S3: Receptor Subtypes, Table S4: Association of rare pathogenic variants (PVs) and a polygenic risk score (PRS) with prevalent breast cancer risk in 6339 older women, Table S5: Per-gene odds ratios (ORs) for prevalent BC risk in pathogenic variant carriers, Table S6: Association of rare pathogenic variants (PVs) and a polygenic risk score (PRS) with prevalent breast cancer risk, stratified by age at diagnosis, Table S7: Chi-squared test $\left(\chi^{2}=1.97, \mathrm{df}=2, p=0.37\right)$. Table S8. List of 313 variants in the PRS, indicating the 271 variants included in the final analysis. 
Author Contributions: P.L.: Conceptualization, Data curation, Funding acquisition, Methodology, Supervision, Writing—original draft. A.B.: Data curation, Formal Analysis, Investigation, Software. M.R.: Conceptualization, Data curation, Formal Analysis, Investigation, Software. S.G.O.: Resources, Data curation, Investigation, Project administration. J.T.: Investigation, Writing—original draft. J.T.N.: Data curation, Formal Analysis, Investigation. P.R.C.: Data curation, Formal Analysis, Investigation. A.D.J.: Conceptualization, Methodology. Y.C.: Conceptualization, Methodology. E.T.W.: Conceptualization, Validation. A.M.: Methodology, Validation. T.N.-D.: Conceptualization, Validation. M.C.S.: Conceptualization, Validation. R.L.M.: Conceptualization, Validation. L.F.: Conceptualization, Data curation. R.S.: Conceptualization, Resources, Data curation, Methodology. E.S.: Conceptualization, Resources. L.G.: Data curation, Methodology. P.G.: Conceptualization, Resources, Data curation, Methodology. B.A.T.: Resources, Data curation, Formal Analysis, Methodology. F.A.M.: Conceptualization, Data curation. P.J.: Conceptualization, Data curation, Resources. I.W.: Conceptualization, Resources, Data curation. C.M.: Conceptualization, Resources, Data curation, Methodology. J.R.Z.: Conceptualization, Resources, Methodology. R.L.W.: Conceptualization, Resources, Methodology. A.T.C.: Resources, Funding acquisition, Project administration, Supervision. A.M.M.: Resources, Funding acquisition, Project administration. J.J.M.: Conceptualization, Resources, Project administration, Supervision. All authors were involved in writing (review and editing). All authors have read and agreed to the published version of the manuscript.

Funding: This work was supported by an ASPREE Flagship cluster grant (including the Commonwealth Scientific and Industrial Research Organisation, Monash University, Menzies Research Institute, Australian National University, University of Melbourne); and grants (U01AG029824 and U19AG062682) from the National Institute on Aging and the National Cancer Institute at the National Institutes of Health, by grants (334047 and 1127060) from the National Health and Medical Research Council of Australia, and by Monash University and the Victorian Cancer Agency. AEC receives an NHMRC Career Development Fellowship (1147843). HPS holds an NHMRC MRFF Next Generation Clinical Researchers Program Practitioner Fellowship (APP1137127). ATC is supported by an NCI Outstanding Investigator Award (R35 CA253185) and is a Stuart and Suzanne Steele MGH Research Scholar. PL is supported by a National Heart Foundation Future Leader Fellowship (ID 102604).

Institutional Review Board Statement: The study received approval from the Alfred Hospital Research Ethics Committee (Project 390/15, 27 August 2015). The ASPREE trial is registered (NCT01038583) and all institutions involved in human participant research received local institutional review board approvals.

Informed Consent Statement: All participants provided written informed consent for genetic research.

Data Availability Statement: The data that support the findings of this study are available from the corresponding author upon request.

Acknowledgments: We thank the trial staff in Australia and the United States, the participants who volunteered for this trial, and the general practitioners and staff of the medical clinics who cared for the participants.

Conflicts of Interest: The authors declare no conflict of interest.

\section{References}

1. Mavaddat, N.; Michailidou, K.; Dennis, J.; Lush, M.; Fachal, L.; Lee, A.; Tyrer, J.P.; Chen, T.H.; Wang, Q.; Bolla, M.K.; et al. Polygenic Risk Scores for Prediction of Breast Cancer and Breast Cancer Subtypes. Am. J. Hum. Genet. 2019, 104, 21-34. [CrossRef]

2. Lakeman, I.M.M.; Rodriguez-Girondo, M.; Lee, A.; Ruiter, R.; Stricker, B.H.; Wijnant, S.R.A.; Kavousi, M.; Antoniou, A.C.; Schmidt, M.K.; Uitterlinden, A.G.; et al. Validation of the BOADICEA model and a 313-variant polygenic risk score for breast cancer risk prediction in a Dutch prospective cohort. Genet. Med. 2020, 22, 1803-1811. [CrossRef]

3. Kapoor, P.M.; Mavaddat, N.; Choudhury, P.P.; Wilcox, A.N.; Lindstrom, S.; Behrens, S.; Michailidou, K.; Dennis, J.; Bolla, M.K.; Wang, Q.; et al. Combined associations of a polygenic risk score and classical risk factors with breast cancer risk. J. Natl. Cancer Inst. 2020, 113, 329-337. [CrossRef] [PubMed]

4. Mars, N.; Koskela, J.T.; Ripatti, P.; Kiiskinen, T.T.J.; Havulinna, A.S.; Lindbohm, J.V.; Ahola-Olli, A.; Kurki, M.; Karjalainen, J.; Palta, P.; et al. Polygenic and clinical risk scores and their impact on age at onset and prediction of cardiometabolic diseases and common cancers. Nat. Med. 2020, 26, 549-557. [CrossRef]

5. Michailidou, K.; Lindstrom, S.; Dennis, J.; Beesley, J.; Hui, S.; Kar, S.; Lemacon, A.; Soucy, P.; Glubb, D.; Rostamianfar, A.; et al. Association analysis identifies 65 new breast cancer risk loci. Nature 2017, 551, 92-94. [CrossRef] 
6. Anglian Breast Cancer Study Group. Prevalence and penetrance of BRCA1 and BRCA2 mutations in a population-based series of breast cancer cases. Br. J. Cancer 2000, 83, 1301-1308. [CrossRef] [PubMed]

7. Kuchenbaecker, K.B.; Hopper, J.L.; Barnes, D.R.; Phillips, K.A.; Mooij, T.M.; Roos-Blom, M.J.; Jervis, S.; van Leeuwen, F.E.; Milne, R.L.; Andrieu, N.; et al. Risks of Breast, Ovarian, and Contralateral Breast Cancer for BRCA1 and BRCA2 Mutation Carriers. JAMA 2017, 317, 2402-2416. [CrossRef] [PubMed]

8. Mavaddat, N.; Peock, S.; Frost, D.; Ellis, S.; Platte, R.; Fineberg, E.; Evans, D.G.; Izatt, L.; Eeles, R.A.; Adlard, J.; et al. Cancer risks for BRCA1 and BRCA2 mutation carriers: Results from prospective analysis of EMBRACE. J. Natl. Cancer Inst. 2013, 105, 812-822. [CrossRef]

9. Barnes, D.R.; Rookus, M.A.; McGuffog, L.; Leslie, G.; Mooij, T.M.; Dennis, J.; Mavaddat, N.; Adlard, J.; Ahmed, M.; Aittomaki, K.; et al. Polygenic risk scores and breast and epithelial ovarian cancer risks for carriers of BRCA1 and BRCA2 pathogenic variants. Genet. Med. 2020, 22, 1653-1666. [CrossRef]

10. Kuchenbaecker, K.B.; McGuffog, L.; Barrowdale, D.; Lee, A.; Soucy, P.; Dennis, J.; Domchek, S.M.; Robson, M.; Spurdle, A.B.; Ramus, S.J.; et al. Evaluation of Polygenic Risk Scores for Breast and Ovarian Cancer Risk Prediction in BRCA1 and BRCA2 Mutation Carriers. J. Natl. Cancer Inst. 2017, 109, djw302. [CrossRef]

11. Lee, A.J.; Cunningham, A.P.; Tischkowitz, M.; Simard, J.; Pharoah, P.D.; Easton, D.F.; Antoniou, A.C. Incorporating truncating variants in PALB2, CHEK2, and ATM into the BOADICEA breast cancer risk model. Genet. Med. 2016, 18, 1190-1198. [CrossRef]

12. Breast Cancer Association Consortium; Dorling, L.; Carvalho, S.; Allen, J.; Gonzalez-Neira, A.; Luccarini, C.; Wahlstrom, C.; Pooley, K.A.; Parsons, M.T.; Fortuno, C.; et al. Breast Cancer Risk Genes-Association Analysis in More than 113,000 Women. N. Engl. J. Med. 2021, 384, 428-439. [CrossRef] [PubMed]

13. Hu, C.; Hart, S.N.; Gnanaolivu, R.; Huang, H.; Lee, K.Y.; Na, J.; Gao, C.; Lilyquist, J.; Yadav, S.; Boddicker, N.J.; et al. A Population-Based Study of Genes Previously Implicated in Breast Cancer. N. Engl. J. Med. 2021, 384, 440-451. [CrossRef] [PubMed]

14. Bahcall, O. Common variation and heritability estimates for breast, ovarian and prostate cancers. Nat. Genet. 2013, 10, 304 . [CrossRef]

15. McNeil, J.J.; Wolfe, R.; Woods, R.L.; Tonkin, A.M.; Donnan, G.A.; Nelson, M.R.; Reid, C.M.; Lockery, J.E.; Kirpach, B.; Storey, E.; et al. Effect of Aspirin on Cardiovascular Events and Bleeding in the Healthy Elderly. N. Engl. J. Med. 2018, 379, 1509-1518. [CrossRef] [PubMed]

16. McNeil, J.J.; Woods, R.L.; Nelson, M.R.; Reid, C.M.; Kirpach, B.; Wolfe, R.; Storey, E.; Shah, R.C.; Lockery, J.E.; Tonkin, A.M.; et al. Effect of Aspirin on Disability-free Survival in the Healthy Elderly. N. Engl. J. Med. 2018, 379, 1499-1508. [CrossRef] [PubMed]

17. McNeil, J.J.; Nelson, M.R.; Woods, R.L.; Lockery, J.E.; Wolfe, R.; Reid, C.M.; Kirpach, B.; Shah, R.C.; Ives, D.G.; Storey, E.; et al. Effect of Aspirin on All-Cause Mortality in the Healthy Elderly. N. Engl. J. Med. 2018, 379, 1519-1528. [CrossRef]

18. ASPREE Investigator Group. Study design of ASPirin in Reducing Events in the Elderly (ASPREE): A randomized, controlled trial. Contemp. Clin. Trials 2013, 36, 555-564. [CrossRef]

19. Nelson, M.R.; Reid, C.M.; Ames, D.A.; Beilin, L.J.; Donnan, G.A.; Gibbs, P.; Johnston, C.I.; Krum, H.; Storey, E.; Tonkin, A.; et al. Feasibility of conducting a primary prevention trial of low-dose aspirin for major adverse cardiovascular events in older people in Australia: Results from the ASPirin in Reducing Events in the Elderly (ASPREE) pilot study. Med. J. Aust. 2008, 189, 105-109. [CrossRef]

20. Lockery, J.E.; Collyer, T.A.; Abhayaratna, W.P.; Fitzgerald, S.M.; McNeil, J.J.; Nelson, M.R.; Orchard, S.G.; Reid, C.; Stocks, N.P.; Trevaks, R.E.; et al. Recruiting general practice patients for large clinical trials: Lessons from the Aspirin in Reducing Events in the Elderly (ASPREE) study. Med. J. Aust. 2019, 210, 168-173. [CrossRef]

21. McNeil, J.J.; Woods, R.L.; Nelson, M.R.; Murray, A.M.; Reid, C.M.; Kirpach, B.; Storey, E.; Shah, R.C.; Wolfe, R.S.; Tonkin, A.M.; et al. Baseline Characteristics of Participants in the ASPREE (ASPirin in Reducing Events in the Elderly) Study. J Gerontol. A Biol. Sci. Med. Sci. 2017, 72, 1586-1593. [CrossRef]

22. Genomes Project Consortium; Auton, A.; Brooks, L.D.; Durbin, R.M.; Garrison, E.P.; Kang, H.M.; Korbel, J.O.; Marchini, J.L.; McCarthy, S.; McVean, G.A.; et al. A global reference for human genetic variation. Nature 2015, 526, 68-74.

23. Taliun, D.; Harris, D.N.; Kessler, M.D.; Carlson, J.; Szpiech, Z.A.; Torres, R.; Taliun, S.A.G.; Corvelo, A.; Gogarten, S.M.; Kang, H.M.; et al. Sequencing of 53,831 diverse genomes from the NHLBI TOPMed Program. Nature 2021, 590, $290-299$. [CrossRef]

24. Lacaze, P.; Sebra, R.; Riaz, M.; Tiller, J.; Revote, J.; Phung, J.; Parker, E.J.; Orchard, S.G.; Lockery, J.E.; Wolfe, R.; et al. Medically actionable pathogenic variants in a population of 13,131 healthy elderly individuals. Genet. Med. 2020, 22, 1883-1886. [CrossRef]

25. Landrum, M.J.; Lee, J.M.; Riley, G.R.; Jang, W.; Rubinstein, W.S.; Church, D.M.; Maglott, D.R. ClinVar: Public archive of relationships among sequence variation and human phenotype. Nucleic Acids Res. 2014, 42, D980-D985. [CrossRef] [PubMed]

26. Lek, M.; Karczewski, K.J.; Minikel, E.V.; Samocha, K.E.; Banks, E.; Fennell, T.; O’Donnell-Luria, A.H.; Ware, J.S.; Hill, A.J.; Cummings, B.B.; et al. Analysis of protein-coding genetic variation in 60,706 humans. Nature 2016, 536, 285-291. [CrossRef] [PubMed]

27. Richards, S.; Aziz, N.; Bale, S.; Bick, D.; Das, S.; Gastier-Foster, J.; Grody, W.W.; Hegde, M.; Lyon, E.; Spector, E.; et al. Standards and guidelines for the interpretation of sequence variants: A joint consensus recommendation of the American College of Medical Genetics and Genomics and the Association for Molecular Pathology. Genet. Med. 2015, 17, 405-424. [CrossRef] [PubMed] 
28. Orchard, S.G.; Lockery, J.E.; Gibbs, P.; Polekhina, G.; Wolfe, R.; Zalcberg, J.; Haydon, A.; McNeil, J.J.; Nelson, M.R.; Reid, C.M.; et al. Cancer history and risk factors in healthy older people enrolling in the ASPREE clinical trial. Contemp. Clin. Trials 2020,96, 106095. [CrossRef]

29. Song, M.; Kraft, P.; Joshi, A.D.; Barrdahl, M.; Chatterjee, N. Testing calibration of risk models at extremes of disease risk. Biostatistics 2015, 16, 143-154. [CrossRef] [PubMed]

30. DeLong, E.R.; DeLong, D.M.; Clarke-Pearson, D.L. Comparing the areas under two or more correlated receiver operating characteristic curves: A nonparametric approach. Biometrics 1988, 44, 837-845. [CrossRef]

31. Sun, X.; Xu, W. Fast Implementation of DeLong's Algorithm for Comparing the Areas under Correlated Receiver Operating Characteristic Curves. IEEE Signal Process. Lett. 2014, 21, 1389-1393. [CrossRef]

32. Fahed, A.C.; Wang, M.; Homburger, J.R.; Patel, A.P.; Bick, A.G.; Neben, C.L.; Lai, C.; Brockman, D.; Philippakis, A.; Ellinor, P.T.; et al. Polygenic background modifies penetrance of monogenic variants for tier 1 genomic conditions. Nat. Commun. 2020, 11, 3635. [CrossRef]

33. Sudlow, C.; Gallacher, J.; Allen, N.; Beral, V.; Burton, P.; Danesh, J.; Downey, P.; Elliott, P.; Green, J.; Landray, M.; et al. UK biobank: An open access resource for identifying the causes of a wide range of complex diseases of middle and old age. PLoS Med. 2015, 12, e1001779. [CrossRef] [PubMed]

34. Stovitz, S.D.; Banack, H.R.; Kaufman, J.S. "Depletion of the susceptibles" taught through a story, a table and basic arithmetic. BMJ Evid. Based Med. 2018, 23, 199. [CrossRef] [PubMed]

35. Khera, A.V.; Chaffin, M.; Aragam, K.G.; Haas, M.E.; Roselli, C.; Choi, S.H.; Natarajan, P.; Lander, E.S.; Lubitz, S.A.; Ellinor, P.T.; et al. Genome-wide polygenic scores for common diseases identify individuals with risk equivalent to monogenic mutations. Nat. Genet. 2018, 50, 1219-1224. [CrossRef] [PubMed]

36. Lee, A.; Mavaddat, N.; Wilcox, A.N.; Cunningham, A.P.; Carver, T.; Hartley, S.; Babb de Villiers, C.; Izquierdo, A.; Simard, J.; Schmidt, M.K.; et al. BOADICEA: A comprehensive breast cancer risk prediction model incorporating genetic and nongenetic risk factors. Genet. Med. 2019, 21, 1708-1718. [CrossRef] [PubMed] 


\section{University Library}

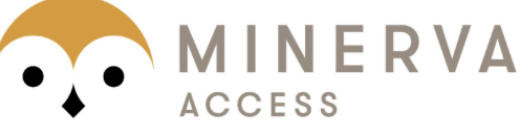

A gateway to Melbourne's research publications

Minerva Access is the Institutional Repository of The University of Melbourne

\section{Author/s:}

Lacaze, P;Bakshi, A;Riaz, M;Orchard, SG;Tiller, J;Neumann, JT;Carr, PR;Joshi, AD;Cao, Y;Warner, ET;Manning, A;Nguyen-Dumont, T;Southey, MC;Milne, RL;Ford, L;Sebra, R;Schadt, E;Gately, L;Gibbs, P;Thompson, BA;Macrae, FA;James, P;Winship, I;McLean, C;Zalcberg, JR;Woods, RL;Chan, AT;Murray, AM;McNeil, JJ

Title:

Genomic Risk Prediction for Breast Cancer in Older Women

\section{Date:}

2021-07-01

\section{Citation:}

Lacaze, P., Bakshi, A., Riaz, M., Orchard, S. G., Tiller, J., Neumann, J. T., Carr, P. R., Joshi, A. D., Cao, Y., Warner, E. T., Manning, A., Nguyen-Dumont, T., Southey, M. C., Milne, R. L., Ford, L., Sebra, R., Schadt, E., Gately, L., Gibbs, P. ,... McNeil, J. J. (2021). Genomic Risk Prediction for Breast Cancer in Older Women. CANCERS, 13 (14), https://doi.org/10.3390/ cancers13143533.

Persistent Link:

http://hdl.handle.net/11343/287416

License:

CC BY 